\title{
STATIONARY MEASURES FOR BRANCHING PROCESSES
}

\author{
J. F. C. KINGMAN
}

Consider a Galton-Watson branching process of the type studied in [1]. The generating function of the family size distribution will be denoted by

$$
f(s)=p_{0}+p_{1} s+p_{2} s^{2}+\cdots,
$$

and we shall assume without further comment that $0<p_{0}<1$. Harris $[1, \S 11]$ has shown that the process admits a stationary measure, i.e., a set of non-negative numbers $\pi_{j}(j=1,2, \cdots)$ satisfying

$$
\pi_{j}=\sum_{i=1}^{\infty} \pi_{i} P_{i j} \quad(j=1,2, \cdots),
$$

where $P_{i j}$ is the probability that, if there are $i$ individuals in one generation, there are $j$ in the next. He has also shown that, if $\left\{\pi_{j}\right\}$ is any non-negative solution of (1), the generating function

$$
\pi(s)=\sum_{j=1}^{\infty} \pi_{j} s^{j}
$$

exists in $|s|<q$ (where $q$ is the probability of extinction), and that, if $\left\{\pi_{j}\right\}$ is normalised so that

$$
\pi\left(p_{0}\right)=1,
$$

then $\pi(s)$ satisfies Abel's functional equation

$$
\pi[f(s)]=\pi(s)+1 \quad(|s|<q) .
$$

Conversely, if a solution $\pi(s)$ of (4) admits a power-series expansion (2) with non-negative coefficients, then the $\pi_{j}$ form a stationary measure.

Harris has conjectured that the stationary measure for any Galton-Watson process is unique up to a constant multiplicative factor, or, equivalently, that there is exactly one stationary measure satisfying (3). The purpose of this note is to provide a counterexample to this conjecture.

Let $\omega$ be any entire function, not identically zero, which has period 1 , and satisfies $\omega(0)=0$. It follows from a well-known property of the equation (4) first noticed by Abel (cf. $[1, \S 11.4]$ ) that, if $\left\{\pi_{j}\right\}$ is a

Received by the editors November 14, 1963. 
stationary measure, normalised by (3), and if $a$ is any real number, then the function $\pi^{a}(s)$ given by

$$
\pi^{a}(s)=\pi(s)+a \omega[\pi(s)]
$$

satisfies (4). Moreover, $\omega[\pi(s)]$ admits a power-series expansion

$$
\omega[\pi(s)]=\sum_{j=1}^{\infty} \chi_{j} s^{j}
$$

in $|s|<q$. If it should happen that

$$
\chi_{j}=O\left(\pi_{j}\right),
$$

then, for all sufficiently small $a$, the coefficients $\pi_{j}+a \chi_{j}$ in the expansion of $\pi^{a}(s)$ are non-negative, and so form a normalised stationary measure which, for $a \neq 0$, is distinct from $\left\{\pi_{j}\right\}$. Thus, if (6) holds, then the stationary measure is not unique (even up to constant multiples).

Now consider the simple case

$$
f(s)=q /(1+q-s),
$$

where $q<1$ is the probability of extinction and the mean family size is $m=1 / q>1$. For this process a normalised stationary measure is given by

$$
\pi_{j}=\left(m^{j}-1\right) / j \log m
$$

with

$$
\pi(s)=\log \left(\frac{1-s}{1-m s}\right) / \log m
$$

Then

$$
\chi_{j}=\frac{1}{2 \pi i} \int \omega\left\{c \log \left(\frac{1-s}{1-m s}\right)\right\} \frac{d s}{s^{j+1}},
$$

where $c=1 / \log m$ and the contour of integration goes once (anticlockwise) round the origin in $|s|<q$. The integrand is single-valued and regular in the closed complex plane cut along a straight line from $q$ to 1 . Moreover, since the imaginary part of $\log [(1-s) /(1-m s)]$ is bounded in this region, and since $\omega$ has a real period, the function

$$
\omega\{c \log [(1-s) /(1-m s)]\}
$$

is bounded. Hence we may deform the contour of integration into 
one which goes from $q$ to 1 above the cut and from 1 to $q$ below it. Then

$$
\chi_{j}=\frac{1}{2 \pi i} \int_{a}^{1} \Omega(x) \frac{d x}{x^{j+1}},
$$

where

$$
\begin{aligned}
\Omega(x)= & \omega\{c \log [(1-x) /(m x-1)]-i c \pi\} \\
& -\omega\{c \log [(1-x) /(m x-1)]+i c \pi\}
\end{aligned}
$$

is bounded. Hence, if $|\Omega(x)| \leqq M$,

$$
\left|\chi_{j}\right| \leqq \frac{M}{2 \pi} \int_{q}^{1} \frac{d x}{x^{j+1}}=\frac{M}{2 \pi} \pi_{j},
$$

so that (6) is satisfied.

Thus, when $f(s)$ is given by (7), the stationary measure is not unique (even up to constant multiples). In fact, we can say more than this. Since, for any integer $k$, an admissible choice of $\omega(s)$ is $\sin (2 \pi k s)$, it follows that the convex cone of stationary measures is infinitedimensional.

A similar result can be proved for any generating function $f(s)$ of the "fractional linear" type in which the mean family size $m$ is not equal to 1 . This is in contrast to the case $m=1$, for which it is proved in [1] that the (normalised) stationary measure is unique.

I am grateful to Dr. T. E. Harris for allowing me to see parts of [1] prior to publication.

\section{REFERENCE}

1. T. E. Harris, The theory of branching processes, Springer, Berlin, 1963.

\section{UnIVERSity OF CAMbridge}

